\title{
Minadores de hojas del aguacate (Lepidoptera: Gracillariidae). Estado actual, Identificación, evaluación de daño y propuesta de manejo
}

\section{Leaf miners of avocado (Lepidoptera: Gracillariidae). Status, identification, damage evaluation and management proposal}

\author{
Francisco Javier Posada-Flórez ${ }^{1 *}$; Helber Adrián Arévalo-Maldonado ${ }^{2}$
}

${ }^{1}$ Ing. Agrónomo, Ph.D. Consultor independiente, Servicios de consultoría y capacitación en laboratorio y en campo en entomología, apicultura y control biológico con énfasis en hongos entomopatógenos. Estados Unidos; e-mail: fjavierposada@gmail.com; (iD) https://orcid.org/0000-0002-7349-2240

${ }^{2}$ Ing. Agrónomo, M.Sc., Ph.D. Universidad de Ciencias Aplicadas y Ambientales, Ingeniería Agronómica, Grupo de Investigación Producción Agrícola Sostenible. Bogotá, Colombia; e-mail: helarevalo@udca.edu.co; (iD) https://orcid.org/0000-0003-4401-452X

*autor para correspondencia: fjavierposada@gmail.com

Cómo citar: Posada-Flórez, F.J.; Arévalo-Maldonado, H.A. 2021. Minadores de hojas del aguacate (Lepidoptera: Gracillariidae). Estado actual, Identificación, evaluación de daño y propuesta de manejo. Rev. U.D.C.A Act. \& Div. Cient. 24(2):e2085. http://doi.org/10.31910/rudca. v24.n2.2021.2085

Artículo de acceso abierto publicado por Revista U.D.C.A Actualidad \& Divulgación Científica, bajo una Licencia Creative Commons CC BY-NC 4.0

Publicación oficial de la Universidad de Ciencias Aplicadas y Ambientales U.D.C.A, Institución de Educación Superior Acreditada de Alta Calidad por el Ministerio de Educación Nacional.

Recibido: julio 27 de 2021

Aceptado: noviembre 11 de 2021

Editado por: Rosângela Brito

\section{RESUMEN}

El cultivo de aguacate representa uno de los principales productos agrícolas de Colombia. Durante las últimas dos décadas, el área sembrada se quintuplicó y se ampliaron, notablemente, las zonas de siembra, en especial, para el cultivar Hass. Con el crecimiento de su producción, también se reportan nuevos problemas fitosanitarios, mayormente, por la adopción de sistemas productivos de otras latitudes del mundo, donde no se cuentan con ecosistemas con presencia de lauráceas y otras plantas relacionadas con el aguacate. Dentro de las especies plagas, se registra a los minadores de hojas del orden Lepidoptera, que se caracterizan por desarrollarse y alimentarse dentro de las hojas, cuyo efecto perjudicial, se genera, en principio, cuando son afectadas las poblaciones de sus enemigos naturales. El primer registro documentado de estos insectos en el país, se realizó a partir de muestras colectadas en un cultivo de aguacate cv. Hass, en Villamaría, (Caldas), que presentó una explosión de las poblaciones de minadores, como consecuencia de la aplicación calendario de plaguicidas, con el criterio de proteger de plagas al cultivo, como arañita roja y trips. Con el fin de evitar que se aumente la frecuencia de eventos, como el enunciado, se desarrolló el trabajo actual, el cual, tuvo como objeto presentar el estado actual de los minadores de hoja del aguacate en Colombia, identificación y descripción del daño del minador de hojas del aguacate en serpentina y en ampolla y brindar recomendaciones para su manejo, en las zonas productoras de Colombia.

Palabras clave: Manejo integrado de plagas; Enemigos naturales; Monocultivo; Phyllocnistis perseafolia; Acrocercops sp.; Eulophidae.

\section{ABSTRACT}

Avocado has become one of the most important crops in Colombia. During the last two decades, the area planted with avocado has quintuplicated, and it is widespread across a wide range of high-altitude lands growing in monoculture with Hass variety. 
Posada-Flórez, F.J.; Arévalo-Maldonado, H.A.: minadores del aguacate en Colombia

As the plantation areas increase, new sanitary problems appear, as consequences of the adoption of technical recommendations from other latitudes where the ecosystems do not have laurels and other plants related to avocado. Within the pest species, the leafminers of Lepidoptera are reported, which the main characteristic is that they develop and feed inside the leaves. The damage they cause happens mainly when the population of their natural enemies is affected. The first record of leafminers in Colombia was done from samples collected in one orchard of avocado cv. Hass in Villa Maria, Caldas where a high infestation outbreak happened as a consequence of the pesticide application following calendar spraying to prevent the attack of pests like red spider mites and thrips. The objective of this paper is to present the status of leafminer pest of avocado leaves in Colombia, providing its identification, description of the serpentine and blotch damage, and proposing recommendations for its management. This will inform the scientific community and farmers about the presence of this pest in avocado crop regions in Colombia, contributing to minimize damaging events like the aforementioned.

Keywords: Integrated pest management; Natural enemies; Monoculture; Phyllocnistis perseafolia; Acrocercops sp.; Eulophidae.

\section{INTRODUCCIÓN}

En los inventarios de las plagas del aguacate en Colombia, se tienen registradas 68 especies de insectos, que se alimentan de diferentes partes del árbol (Posada Ochoa, 1989) y dentro, de las cuales, las plagas de mayor importancia económica son los perforadores de fruto y tallo. Adicionalmente, varias especies de trips y el mírido Monalonion velezangeli (Carvalho y Costa, 1988), han cobrado importancia (Cárdenas Murillo \& Posada Flórez, 2001; Londoño, 2020). De igual manera, durante las dos últimas décadas, se aumentó el registro de especies plaga potenciales, entre las que se encuentran, Laurencella colombiana Foldi \& Watson, 2001, (Hemiptera: Monophlebidae), Bruggmanniella perseae Gagné, 2004 (Diptera: Cecidomyiidae) y Heilipus elegans Guérin-Méneville, 1844 (Gagné et al. 2004; Gil P. et al. 2007; Rubio G. et al. 2009). Además, se incrementó el conocimiento de escamas Coccoidea, asociadas al cultivo (Kondo \& Muñoz, 2016).

Colombia es el cuarto productor de aguacate en el mundo, con 211.056 hectáreas (FAO, 2019), razón por la cual, es de permanente interés conocer los insectos asociados a los agroecosistemas aguacateros del país. Mientras no se cuente con esta información, los productores aplicarán modelos de manejo de plagas de otras latitudes, donde no se cuentan con lauráceas y otras plantas relacionadas con el aguacate, desconociendo los servicios ambientales brindados por los ecosistemas circundantes.

Entre los servicios ambientales ofrecidos, se encuentra una gran diversidad de organismos benéficos nativos y con una estrecha relación con las plantas de aguacate (Gil P. et al. 2007). Entre los roles de los organismos benéficos, se pueden resaltar la polinización y el control natural de fitófagos, que garantizan la producción del cultivo; sin embargo, es necesario precisar que estos ecosistemas son muy sensibles, por lo que un manejo inadecuado de plagas y malezas podría desencadenar un desequilibrio ecológico y potencializar el efecto perjudicial de especies plaga.

Entre las plagas reportadas para el aguacate en el nuevo mundo, se encuentran los minadores de hojas Phyllocnistis perseafolia Davis \& Wagner, 2011, P. byperpersea Davis \& Wagner, 2011 y Caloptilia perseae (Busck, 1920); específicamente para Colombia, se reporta a P. perseafolia. Hasta el momento, la mayoría de los reportes indican a los minadores de hojas del aguacate como plagas menores o potenciales; por ejemplo, $C$. persea es considerada una plaga menor en México y en Cuba y, de igual manera, los daños causados por Phyllocnistis sp., en la Florida, Estados Unidos y en Perú, son despreciables (Wysoki et al. 2002).

Los minadores de hoja Gracillariidae, se alimentan y se desarrollan habitualmente dentro de las hojas jóvenes; sin embargo, también se pueden encontrar atacando tallos, frutos o flores ( $\mathrm{Hu}$ et al. 2011). Los rastros generados por los minadores son característicos y permiten su identificación en campo, al menos, hasta nivel de género.

La mayoría de gracilaríidos, se consideran plagas menores o potenciales, debido a que sus poblaciones son reguladas y mantenidas por debajo de los niveles de daño económico, principalmente, por parasitoides de la familia Eulophidae (Yefremova \& Kravchenko, 2015; Kanhar et al. 2017; Lee et al. 2017).

Teniendo en cuenta las razones expuestas, el presente trabajo tuvo como objeto, indicar el estado actual de los minadores de hoja del aguacate en Colombia, reconocer los enemigos naturales, describir el daño y brindar elementos para el manejo de los minadores de hoja, acordes con los agroecosistemas donde se desarrollan los cultivos.

\section{MATERIALES Y MÉTODOS}

Registro de minadores. El registro inicial de los minadores de hoja del aguacate, se realizó a partir de una visita técnica a una plantación de aguacate Hass, para la evaluación de plagas, ubicada en el municipio de Villamaría, Caldas, a una altitud de $2.200 \mathrm{~m}$ s.n.m., dentro de la zona de vida bosque muy húmedo montano bajo (bmh-MB), con una extensión de 10 hectáreas y una densidad de 120 árboles por hectárea. Los reportes adicionales, se presentan a partir de las observaciones de asistentes técnicos de las diferentes zonas productoras de aguacate de Colombia.

Identificación de especies. Durante la visita técnica a la finca de Villamaría, se colectaron 20 hojas por árbol con minas, con daño fresco y daño viejo, para analizarlas en el laboratorio, donde se clasificaron por el tipo de mina y se utilizaron para recuperar adultos de los minadores y posibles enemigos naturales. Después de clasificar las hojas con minas en serpentina y en ampolla, se colocaron en una bolsa plástica cinco hojas con toallas de papel, para colectar el exceso de humedad y evitar que las muestras fueran colonizadas por hongos. Diariamente, las bolsas con las hojas, se 
revisaron para colectar los insectos que emergieron de las minas. Las hojas con minas, se mantuvieron en observación 21 días y antes de descartarlas se revisaron, para establecer que no había presencia de insectos en las minas.

Los especímenes de minadores colectados, se enviaron al Dr. Donald Davis, especialista en Lepidoptera, Gracillariidae (Department of Entomology, National Museum of Natural History, Smithsonian Institution) y el parasitoide fue identificado por Lucia Pérez (Laboratorio de Entomología de la Universidad de la Amazonía).

Evaluación del daño generado por los minadores. La evaluación, se realizó en la plantación de aguacate Hass, que se indicó. En cada lote, se tomaron 12 (10\%) árboles, tanto en desarrollo como en producción. En cada árbol, se hicieron observaciones sobre los problemas de plagas, incluidos los minadores. La presencia del daño de los minadores, se registró para cada árbol, haciendo evaluaciones sobre las hojas de los terminales y frutos. De los árboles evaluados, se tomaron al azar cinco árboles y, en cada uno, se tomó, en los cuatro puntos cardinales, un terminal del estrato bajo y uno del estrato alto, para contar el número de hojas y, de éstas, el número con daño de minadores. Adicionalmente, se hizo una evaluación de frutos, tanto en los árboles $(\mathrm{N}=10)$ como en los cosechados $(\mathrm{N}=100)$, para determinar la presencia de daño de los minadores.

Con los datos de la evaluación de los minadores, se estimó la incidencia del daño en los árboles y en los terminales, mientras que la severidad del daño en las hojas y frutos, se evaluó por el número de hojas o frutos con presencia de los minadores. La incidencia y la severidad, se estimaron con las siguientes fórmulas:

Incidencia de árboles con daño \% = árboles con daño / total árboles evaluados X 100.

Incidencia de terminales con daño $\%=$ terminales con daño $/$ total terminales evaluados X 100.

Severidad del daño en las hojas de los terminales $\%=$ hojas con daño / total hojas evaluadas x 100 .

Severidad del daño en frutos $\%=$ frutos con daño / total frutos evaluadas x 100 .

Además, durante la evaluación del daño, se registraron los métodos de manejo empleados en el cultivo, para complementar la discusión.

\section{RESULTADOS Y DISCUSIÓN}

Registro de minadores. Como se mencionó, el primer registro documentado de minadores de hoja del aguacate en Colombia fue realizado por Davis \& Wagner (2011), a partir de los especímenes colectados en el presente estudio. Entre los minadores encontrados, uno corresponde al minador en serpentina del aguacate $P$. perseafolia $y$, el otro, al minador en ampolla del aguacate pendiente por describir y cuya identificación a género, se presenta más adelante.

Minador de hojas del aguacate en serpentina -MHAS. Los reportes de este minador, por parte de productores, asistentes técnicos y personas del común, aumentaron desde su observación, en el 2008. Los reportes corresponden a árboles de aguacate en cultivo, ecosistemas naturales o sembrados, con fines decorativos. Entre los lugares donde se reporta al MHAS, se encuentran: Antioquia, Caldas (Pacora, Aguadas), Cundinamarca (Bogotá, La Vega), Quindío (Armenia, Pijao, Salento, Calarcá, Génova), Risaralda (Santuario) y Tolima (Cajamarca y Fresno). Posiblemente, en todos los casos, se trate de $P$. perseafolia, debido a la monofagia exhibida por la mayoría de las especies de Phyllocnistis y a que, hasta el momento, en Colombia, es la única especie minadora de este género, reportada para Suramérica; sin embargo, esta premisa no se puede asegurar, debido a que Davis \& Wagner (2011) reportan en aguacate, a $P$. hyperpersea para el nuevo mundo y, además, el gran desconocimiento de Phyllocnistis en la región (Brito et al. 2017), da lugar a que se trate, inclusive, de un complejo de especies.

Adicionalmente, el MHAS, se ha observado en diferentes viveros en Colombia y en México (Senasica, 2018). Es preciso aclarar que, de acuerdo con el daño reportado en México, por Senasica (2018), corresponde a una especie de Phyllocnistis y no a Caloptilia perseae.

Minador de hojas del aguacate en ampolla-MHAA. Hasta el momento, solo se reporta para el cultivo mencionado en el presente trabajo.

\section{Identificación de especies.}

MHAS. A partir de los especímenes colectados de las minas en serpentina, la especie fue descrita por Davis \& Wagner (2011), como P. perseafolia (Figura 1e); estos autores describen el adulto macho y hembra, la pupa y el tipo de mina generado por la larva; no obstante, hace falta la descripción de la larva, tanto en los instares succionadores de sabía como del instar hilador, lo cual, sería de gran ayuda para su identificación, ya que, habitualmente, el estado larval es el que más se encuentra en campo.

MHAA. De acuerdo con el desarrollo, tipo de mina y algunos caracteres del adulto, esta especie corresponde a Acrocercops. Teniendo en cuenta las especies descritas de este género para el neotrópico (De Prins et al. 2019) y la comunicación personal del Dr. Donald Davis, se trata de una nueva especie. Acrocercops no está reportada en lauráceas para el neotrópico y, de acuerdo con el patrón de coloración alar (Figura 2e), corresponde a una especie relacionada con A. cissiella Busck, 1934 y A. maranthaceae Busck, 1934.

Acrocercops y otros géneros de Acrocercopinae y Ornixolinae, se caracterizan por cumplir todo el estado larval dentro de la hoja y generar, inicialmente, una mina en serpentina y, luego, una mina lagunar tentiforme o en ampolla. El último instar larval es de color rojizo, en el cual, abandona la mina y forma el capullo en la misma 


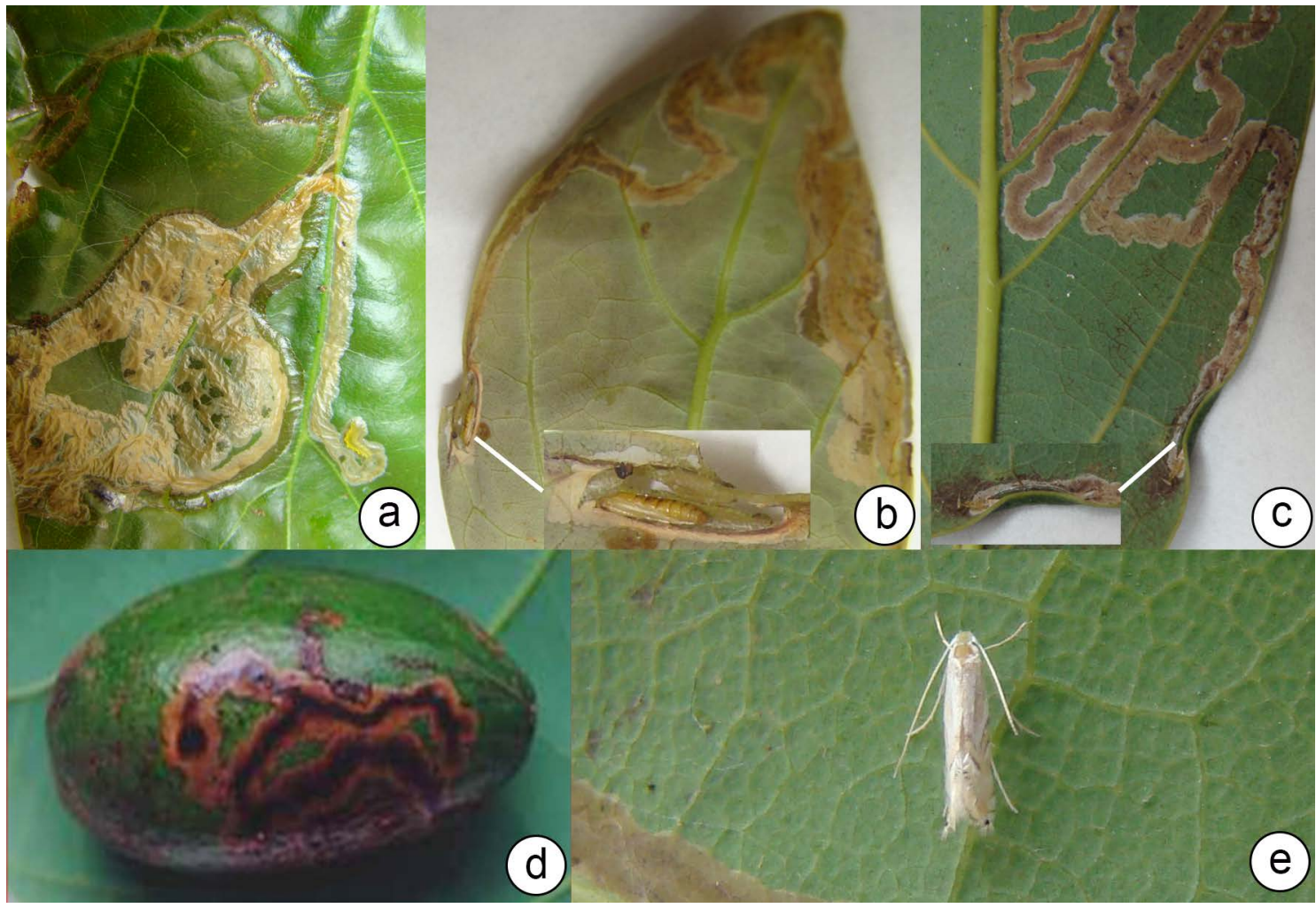

Figura 1. Minador de hojas del aguate en serpentina-MHAS, Phyllocnistis perseafolia. a. Mina del MHAS en el haz de la hoja; b. Mina del MHAS en el envés de la hoja, resaltando la cámara pupal en la margen de la hoja y abierta para mostrar la pupa; c. Cámara pupal con la exuvia de la pupa adherida al orificio de emergencia del adulto; d. Mina del MHAS en fruto; e. Adulto del MHAS.

hoja o en hojas aledañas; el capullo presenta burbujas sobre la superficie (Kumata et al. 1988). Como se observa en las figuras 2ad, todas las características enunciadas, las presenta el MHAA.

En cuanto el adulto, la posición en reposo con la parte anterior levantada de la superficie es característico para las subfamilias Acrocercopinae, Gracillariinae y Ornixolinae (Figura 2e).

Parasitoides. Del MHAS, se obtuvieron 3 morfoespecies de la familia Eulophidae y una corresponde al género Horismenus (Figura 3). En este punto, se recalca la necesidad de realizar trabajos tendientes al reconocimiento y la posible descripción de las especies de parasitoides, asociadas a los minadores del aguacate.

Evaluación del daño generado por los minadores. En el cultivo de Villa María, el daño por los minadores de serpentina y ampolla, se encontró en todos los árboles evaluados, tanto en desarrollo como en producción, causando defoliación y pérdida de área fotosintética. Igualmente, el ataque de los minadores, se registró generalizado en los terminales de todos los estratos de la planta y la incidencia del daño fue del $100 \%$. Por terminal $(n=40)$ de la evaluación, de cinco árboles, en los cuatro puntos cardinales y, en cada punto, un terminal en el estrato alto y, el otro, del estrato bajo, con un promedio de 15 hojas por terminal. Se encontró un promedio de 10 hojas con una severidad de daño del 66,6\%, del MHAS y 3 hojas, con daño del MHAA, con una severidad de daño del $20 \%$. Las hojas, tanto las recién formadas como las desarrolladas, presentaron presencia de minas.

Las minas en serpentina se presentaron, tanto por el haz como en el envés de las hojas (Figuras 1a-c) y, por hoja, se encontró 3,2 $\pm 1,7$ minas en promedio (Min 1, Max 8, N=100) y algunas minas, se mostraban entrecruzadas. Las minas en ampolla, se presentaron sobre el haz (Figura 2a) y evidenció $1,5 \pm 0,8$ minas en promedio (Min. 1, Max. 4, N=100). Estas minas, se presentaban, generalmente individuales, con una área y configuración muy definida de ampolla. Los dos tipos de minas presentes en una misma hoja, se halló en un $15 \%$ de las hojas evaluadas $(\mathrm{N}=100)$.

Las minas de serpentina en el haz, se observaron plateadas, probablemente, por la exposición al sol, mientras cuando se presentaron en el envés, el color era verde o café, tal vez, debido a la oxidación de los tejidos de la epidermis de hojas dañados. El seguimiento de la mina, desde que se inició hasta que terminó en la hoja, demostró la voracidad de la larva, que recorrió toda la extensión de la hoja, consumiendo el tejido debajo de la epidermis (Figuras 1a-c). El último instar larval, se desplaza hacia el margen de la hoja, el cual, dobla hacia la parte atacada, haz o envés, donde forma el capullo y, en cada uno de estos, solo se encontró un espécimen (Figura 1b). P. perseafolia también se presentó atacando frutos (Figura 1d), pero la severidad de este daño fue baja. En los frutos evaluados en los árboles fue de 3,2\% ( $\mathrm{n}=120$ árboles) y en los cosechados de $3 \%$ ( $n=100$ cosechados). En este punto es preciso resaltar que, usualmente, los Phyllocnistis se desarrollan 


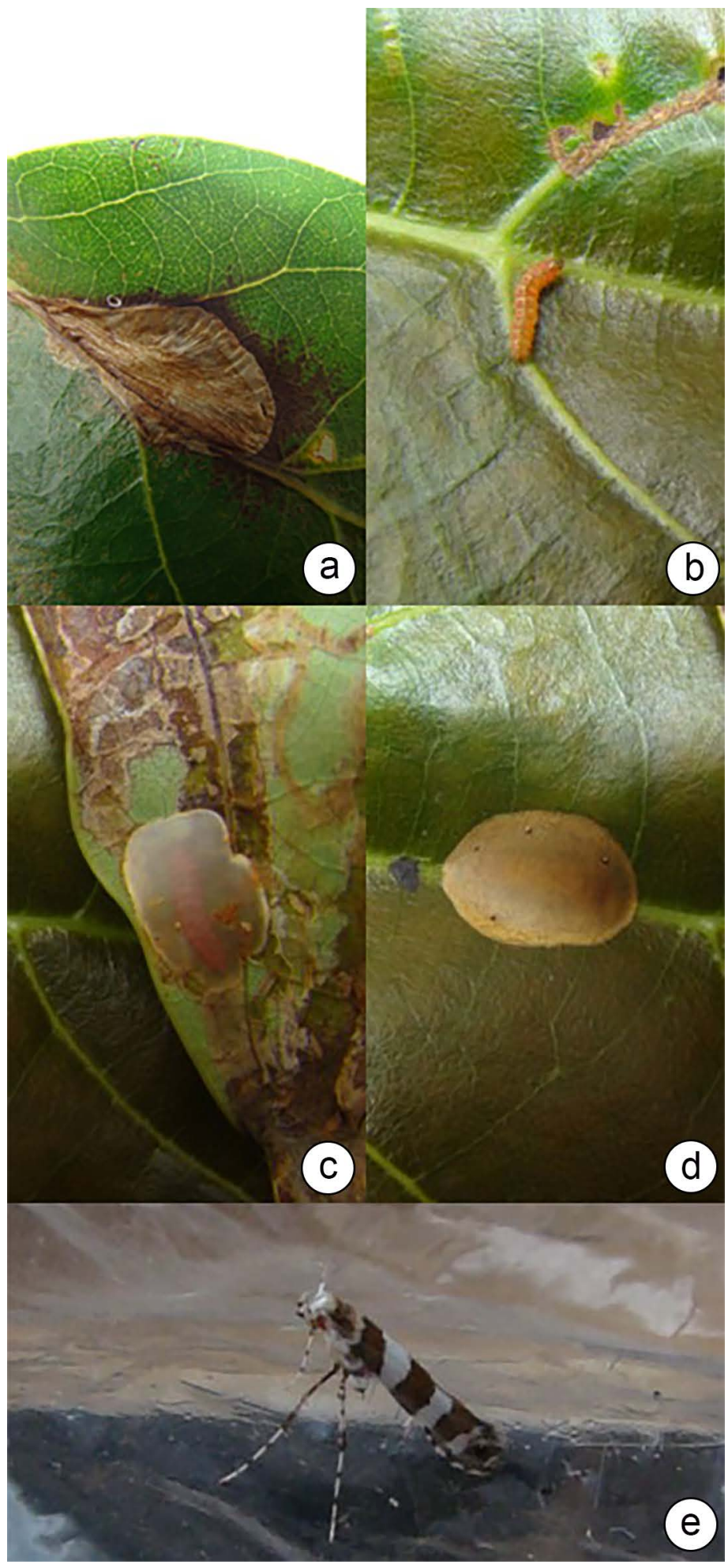

Figura 2. Minador de hojas del aguate en ampolla-MHAA, Acrocercops sp. a. Mina en ampolla en el haz de la hoja; b. Larva de último instar después de emerger para realizar el capullo y empupar fuera de la mina; c. Larva dentro del capullo de seda afuera de la mina; d. Pupa y capullo de forma esférica y semitransparente con cuatro burbujas sobre la superficie; e. adulto en posición de reposo.

en el envés de las hojas jóvenes y un indicio de su abundancia es encontrarlos en el haz de las hojas y en otras estructuras de la planta.
Las minas de ampolla, se presentaron sobre el haz y el daño se pudo evaluar por el envés de la hoja, observando los terminales desde el piso. Estas minas, inicialmente, comenzaron como serpentinas en el lugar de oviposición y después de recorrer unos centímetros, se 


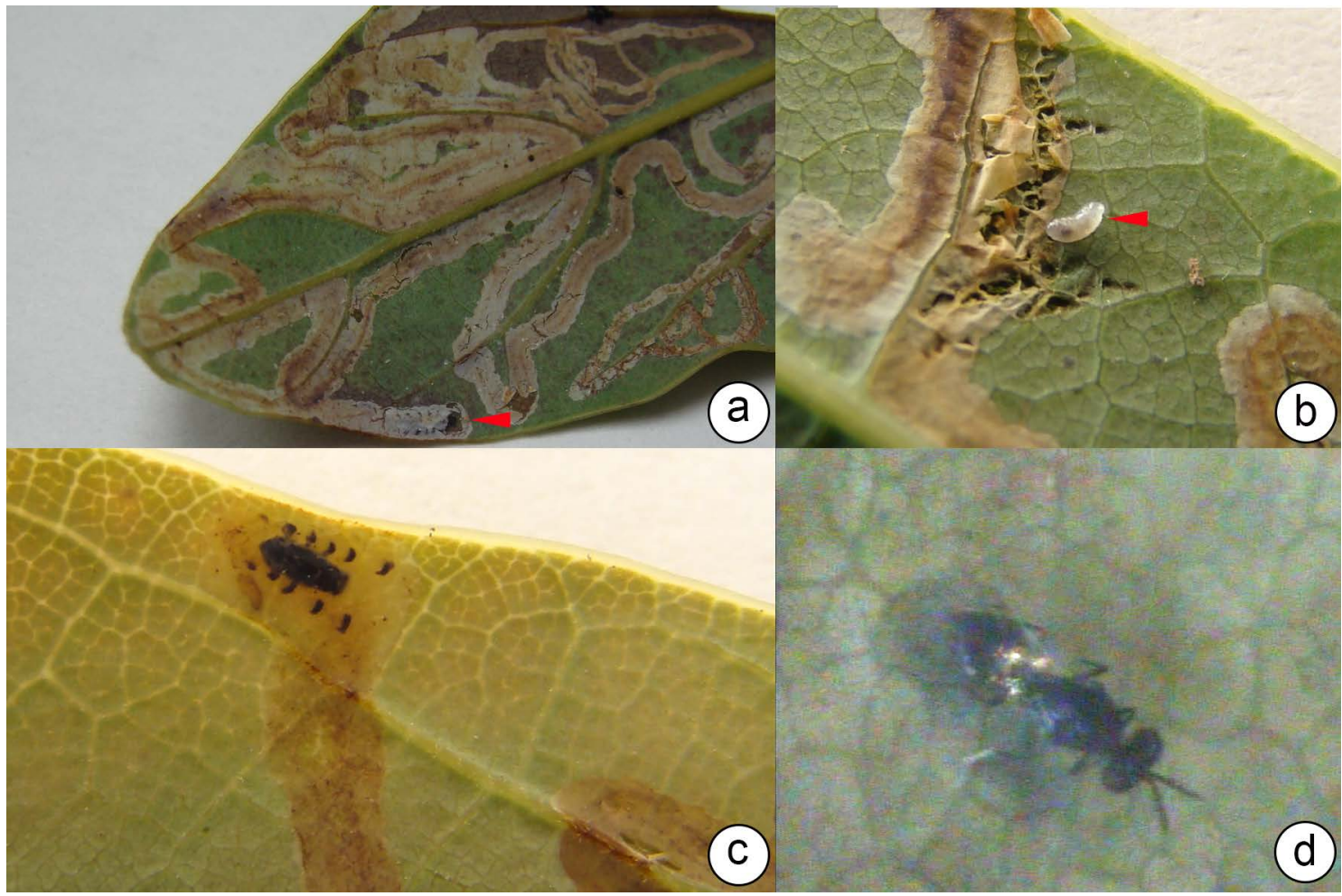

Figura 3. Horismenus sp. (Eulophidae) parasitoide del minador de hojas del aguacate en serpentina -MHAS. a. Hoja con minas mostrando la larva del MHAS atacada por el parasitoide (flecha); b. Larva del parasitoide extraída de la mina (flecha); c. Pupa del parasitoide desarrollándose dentro de la mina del MHAS; d. Adulto del parasitoide que emergió de la larva del MHAS.

formó la mina en ampolla, que se expande en forma radial, por la actividad de alimentación de las larvas. Por ampolla, se encontró un máximo de dos larvas y abundantes depósitos de excrementos de color negro (Figura 2a).

La importancia económica de minadores de serpentina y ampolla encontrados en cultivos de aguacate va a depender del manejo de los cultivos. Los minadores de hojas son un grupo de plagas de difícil manejo, porque permanecen protegidas dentro de las minas (Wysoki et al. 2002). En Colombia y otros países son conocidas las crisis insostenibles causadas por los minadores de hojas, en varios cultivos, como flores, arveja, habichuela, frijol, papa, café, melón, tomate y cítricos (Vélez-Ángel et al. 1982; Zenner de Polania, 1986; León \& Campos, 1999; Cárdenas Murillo \& Posada Flórez, 2001; Constantino et al. 2011). Está demostrado que el daño que causan los minadores de hojas en diferentes cultivos está directamente relacionado con las aplicaciones de plaguicidas, que crean resistencia de los minadores a los insecticidas, matan los enemigos naturales y generan la explosión de las poblaciones de los minadores.

De acuerdo con lo mencionado, probablemente, lo que ocurrió en el cultivo de aguacate Hass, en la finca de Villa María, fue un desequilibrio ecológico, que afecto las poblaciones de enemigos naturales y que condujo a la infestación mencionada, ya que el manejo de plagas en la finca se estaba haciendo semanalmente, siguiendo el criterio de aplicaciones de plaguicidas calendario, para prevenir ataque de las plagas. En los lotes, se observó la aplicación de mezclas de plaguicidas utilizando bombas estacionarias. La evaluación de las aplicaciones de los plaguicidas demostró deriva de la aplicación y bajo cubrimiento de gotas en las hojas de los árboles recién aplicados. Las aplicaciones estaban dirigidas a controlar arañita roja (Acarina: Tetranychidae) y trips (Thysanoptera: Thripidae), pero en las muestras de hojas evaluadas no se encontró presencia de estas plagas, ni del daño.

Este método de manejo de plagas no es técnico, ni sigue las recomendación del manejo integrado de plagas - MIP, que incluye la identificación de los organismos plaga, conocer la dinámica de la población, establecer niveles de infestación, evaluar la severidad, determinar los umbrales de daño, conocer la ecofisiología del cultivo, reconocer la biología del insecto que causa el daño, determinar los factores de mortalidad y reconocer los beneficios y consecuencias de los métodos de control (Wysoki et al. 2002).

En cultivos de frutales, como el aguacate, se sugiere seguir las recomendaciones enunciadas por el MIP, en lugar de hacer aplicaciones de plaguicidas de forma "calendario", con el fin de prevenir la presencia de plagas. Prevenir lo que no se conoce, solo conduce a los desastres ecológicos, causados por el uso indiscriminado de plaguicidas y a generar altos costos de producción; estas prácticas de manejo, con seguridad crearán crisis económicas y no posibilitará continuar con el cultivo, en forma similar, como aconteció para el algodón en Colombia, considerado el "oro blanco" (Federalgodon, 1990). Ahora, el aguacate es considerado el "oro verde", que se puede convertir en otro espejismo, como la búsqueda del "dorado", por los conquistadores. 
El manejo de las poblaciones de minadores en este cultivo, después de los desequilibrios generados por el uso indiscriminado de plaguicidas, solo se pudo lograr fomentando la fauna benéfica, suspendiendo las aplicaciones calendario e implementando programas de MIP. Anecdóticamente, se ha escuchado de los agricultores experimentados, "que para tener plagas de minadores solo se requiere aplicar insecticidas".

A parte de este caso particular, los técnicos y productores no han reportado otro episodio, donde los minadores de hojas del aguacate hayan generado pérdidas económicas; sin embargo, a continuación, se describen una serie de recomendaciones para evitar que se ocasione una explosión de las poblaciones de minadores de hoja del aguacate en el país o en otras zonas productoras del mundo.

-Corroborar las especies de minadores de hojas del aguacate en serpentina en las zonas reportadas en el país y en el nuevo mundo, para asegurar el éxito de la implementación de programas de control biológico, por conservación o por inundación y evitar la diseminación de las diferentes especies de Phyllocnistis, en las regiones productoras de aguacate.

-Vigilar y controlar el movimiento de plantas y frutos, para evitar la diseminación de los minadores de hojas a lugares donde los minadores de hoja no se han reportado y evitar que lleguen a zonas con baja presencia de enemigos naturales.

-Determinar el efecto de los insecticidas sobre los enemigos naturales de los minadores de hoja del aguacate, especialmente, de las especies de eulófidos, que son los principales reguladores de los minadores de hoja del orden Lepidoptera (Yefremova \& Kravchenko, 2015).

-Mantener franjas o áreas con plantas nativas en el cultivo del aguacate, para favorecer las poblaciones de los enemigos naturales de los minadores de hojas del aguacate, ya que los eulófidos $\mathrm{y}$ otros enemigos naturales requieren de refugio $\mathrm{y}$ alimento adicional, al brindado por su hospedante, para aumentar su longevidad, producción de huevos y capacidad de búsqueda (Kishinevsky et al. 2018). Además, esta práctica, a su vez, es favorable para el mantenimiento de los polinizadores nativos, entre los que se encuentra, una gran diversidad de dípteros, coleópteros, himenópteros, en especial, avispas y abejas sin aguijón Trigonas, Meliponas, Bombus o abejas solitarias, que necesitan sitos de anidamiento (Wysoki et al. 2002; Peña, 2003; CastañedaVildózola et al. 1999).

-Además de realizar un uso racional de insecticidas para el control de otras plagas del cultivo de aguacate, se recomienda un uso racional de herbicidas, ya que se ha demostrado que algunos ingredientes activos, como la atrazina, el paraquat y el nicosulfuron, afectan las poblaciones de eulófidos (De Menezes et al. 2014).

-Identificar las especies de eulófidos y otros enemigos naturales, asociados a los minadores de hojas del aguacate, determinar cuáles se podrían usar dentro de un plan de control biológico para las zonas donde las poblaciones de enemigos naturales sea escaza y generar métodos para su cría e implementación.

Un manejo de plagas del aguacate implementando las recomendaciones enunciadas puede llevar a establecer la producción orgánica del cultivo y generar un producto certificado, que puede llegar a tener mayor demanda y mejores precios, en los mercados internacionales.

Es preciso resaltar que el aguacate, al tratarse de una planta perenne, facilita la implementación de sistemas de producción orgánica o con menor uso de insumos, ya que los árboles de aguacate pueden soportar una alta diversidad y abundancia de insectos herbívoros, al presentar defensas físicas y químicas, que los protegen del efecto nocivo de estas especies (Hernández-Cumplido et al. 2021). Aunque es necesario evaluar cómo la domesticación del aguacate ha favorecido las poblaciones de minadores de hojas, ya que la mayoría de los reportes de minadores de hojas del aguacate, corresponden a la variedad Hass.

Agradecimientos. Los autores expresan su agradecimiento a María Camila Ovalle y Daniela Cárdenas Alarcón, asistentes técnicos del cultivo de aguacate y a Martha Eugenia Londoño, productora y asistente técnico de aguacate, quienes brindaron información de la distribución del minador de hojas del aguacate en serpentina. A Lucía Pérez Benavides, especialista en microhimenópteros, quien identificó los parasitoides del MHAS y a Donald Davis, especialista de microlepidópteros, quien describió al MHAS, como Phyllocnistis perseafolia. Conflicto de intereses: El manuscrito fue preparado y revisado por los autores, quienes declaran ser los únicos autores y que no existe ningún conflicto de intereses que ponga en riesgo la validez de los resultados presentados.

\section{REFERENCIAS}

1. BRITO, R.; LOPEZ-VAAMONDE, C.; GONCALVES, G.L.; BECKER, V.O.; MIELKE, O.H.H.; MOREIRA, G.R.P. 2017. Taxonomic revision of Neotropical Phyllocnistis Zeller, 1848 (Lepidoptera: Gracillariidae), with descriptions of seven new species and host plant associations. Zootaxa. 4341(3):301-352. https://doi.org/10.11646/zootaxa.4341.3.1

2. CÁRDENAS MURILLO, R.; POSADA FLÓREZ, F. 2001. Los insectos y otros habitantes de cafetales y platanales. Comité Departamental de Cafeteros del Quindío (Armenia, Colombia). 250p.

3. CASTAÑEDA-VILDÓZOLA, A.; EQUIHUA-MARTÍNEZ, A.; VALDÉS-CARRASCO, J.; BARRIENTOS-PRIEGO, A.F.; ISH-AM, G.; GAZIT, S. 1999. Insectos polinizadores del aguacatero en los estados de México y Michoacán. Revista Chapingo Serie Horticultura. 5:129-136.

4. CONSTANTINO, L.M.; FLÓREZ, J.C.; BENAVIDES, P.; BACCA, T. 2011. Minador de hojas del cafeto. Una plaga 
potencial por efectos del cambio climático. Avances técnicos Cenicafé. 409:1-12.

5. DAVIS, D.R.; WAGNER, D.L. 2011. Biology and systematics of the New World Phyllocnistis Zeller leafminers of the avocado genus Persea (Lepidoptera, Gracillariidae). ZooKeys. 97:39-73.

https://doi.org/10.3897/zookeys.97.753

6. DE MENEZES, C.W.; SOARES, M.A.; FONSECA, A.J.; DOS SANTOS, J.B.; CAMILO, S.D.S.; ZANUNCIO, J.C. 2014. Palmistichus elaeisis (Hymenoptera: Eulophidae) as an indicator of toxicity of herbicides registered for corn in Brazil. Chilean Journal of Agricultural Research. 74(3):361-365.

https://doi.org/10.4067/S0718-58392014000300016

7. DE PRINS, J.; ARÉVALO-MALDONADO, H.A.; DAVIS, D.R.; LANDRY, B.; VARGAS, H.A.; DAVIS, M.M.; BRITO, R.; FOCHEZATO, J.; OHSHIMA, I.; MOREIRA, G.R.P. 2019. An illustrated catalogue of the Neotropical Gracillariidae (Lepidoptera) with new data on primary types. Zootaxa. 4575(1):1-110.

https://doi.org/10.11646/zootaxa.4575.1.1

8. FEDERACION NACIONAL DE ALGODONEROS, FEDERALGODON. 1990. Bases técnicas para el cultivo del algodón en Colombia. Federación Nacional de Algodoneros. División Técnica Bogotá (Colombia). 714p.

9. FOOD AND AGRICULTURE ORGANIZATION, FAO. 2019. FAOSTAT Statistical Database of the United Nation Food and Agriculture Organization (FAO) statistical division. Rome. Disponible desde internet en:

http://www.fao.org/faostat/en/\#search/avocado (Con acceso el 08/08/2021).

10. GAGNÉ, R.J.; POSADA, F; GIL, Z.N. 2004. A new species of Bruggmanniella (Diptera: Cecidomyiidae) aborting young fruit of avocado, Persea americana (Lauraceae), in Colombia and Costa Rica. Proceedings of the Entomological Society of Washington. 106(3):547-553.

11. GIL P., Z.N.; POSADA F., F.J.; PÉREZ S., M.; CÁRDENAS M., R. 2007. Registro y notas bionómicas de una nueva plaga del aguacate Laurencella colombiana (Hemiptera: Margarodidae) en Colombia. Rev. U.D.C.A Act. \& Div. Cient. 10(2):43-50.

http://dx.doi.org/10.31910/rudca.v10.n2.2007.583

12. HERNÁNDEZ-CUMPLIDO, J.; RODRIGUEZ-SAONA, C.; RUÍZ-RODRÍGUEZ， C.E.; GUEVARA-FEFER, P.; AGUIRRE-PALEO, S.; MIRANDA TREJO, S.; CALLEJAS-CHAVERO, A. 2021. Genotypic variation in plant traits, chemical defenses, and resistance against insect herbivores in avocado (Persea americana) across a domestication gradient. Frontiers in Agronomy. 2:1-12.

https://doi.org/10.3389/fagro.2020.616553

13. HU, B; WANG, S.; ZHANG, J.; LI, H. 2011. Taxonomy and biology of two seedparasitic gracillariid moths (Lepidoptera, Gracillariidae), with description of a new species. ZooKeys. 83:43-56.

https://doi.org/10.3897/zookeys.83.783

14. KANHAR, K.A.; KANHER, F.M.; PANHWAR, R.; TUNIO, S.A.; SHAIKH, A.M.; HASSAN AWAN, R.R. 2017. Parasitoids associated with mango leaf miner, Acrocercops syngramma (Meyrick) Lepidoptera: Gracillariidae in Mango Orchard. Journal of Entomology and Zoology Studies. 5(4):1582-1588.

15. KISHINEVSKY, M.; COHEN, N.; CHIEL, E.; WAJNBERG, E.; KEASAR, T. 2018. Sugar feeding of parasitoids in an agroecosystem: effects of community composition, habitat and vegetation. Insect Conservation and Diversity. 11(1):50-57. https://doi.org/10.1111/icad.12259

16. KONDO, T.; MUÑOZ, J.A. 2016. Scale insects (Hemiptera: Coccoidea) associated with avocado crop, Persea americana Mill. (Lauraceae) in Valle del Cauca and neighboring departments of Colombia. Insecta Mundi. 465:1-24.

17. KUMATA, T.; KUROKO, H.; ERMOLAEV, V.P. 1988. Japanese species of the Acrocercops-group (Lepidoptera: Gracillariidae) Part I. Insecta Matsumurana. Series Entomology. New Series. 38:1-111.

18. LEE, S.; KIM, D.S.; KIM, I.K.; CHOI, C.W.; HWANG, R.; KU, D.S.; BYUN, B.K. 2017. Indigenous parasitoids as effective natural enemies of Phyllocnistis citrella (Lepidoptera: Gracillariidae) in Korea. Journal of Forestry Research. 28(1):183-187.

https://doi.org/10.1007/s11676-016-0304-4

19. LEÓN, G.; CAMPOS, J. 1999. Fluctuación poblacional del minador de los cítricos Pbyllocnistis citrella (Lepidoptera: Gracillariidae) en el piedemonte del departamento del Meta. Revista Colombiana de Entomología. 25(3-4):147-150.

20. LONDOÑO, M.E. 2020. Manejo integrado de Monalonion velezangeli en aguacate. $2 \mathrm{da}$ reimpresión. Agrosavia. Mosquera, Colombia. 20p. Disponible desde Internet en: https://repository.agrosavia.co/bitstream/handle/20.500.1 2324/1931/Ver_Documento_1931pdf?sequence=1\&isAllo wed $=y($ con acceso el 01/07/2021)

21. PEÑA, J.E. 2003. Insectos polinizadores de frutales tropicales: no solo las abejas llevan la miel al panal. Manejo Integrado de Plagas y Agroecología (Costa Rica). 69:6-20. 
22. POSADA OCHOA, L.M. 1989. Lista de insectos dañinos y otras plagas en Colombia (No. L-0071). Instituto Colombiano Agropecuario, ICA.

23. RUBIO G., J.D.; POSADA F., F.J.; OSORIO L., Ó.I.; VALLEJO E., L.F.; LÓPEZ N., J.C. 2009. Primer registro de Heilipus elegans Guérin-Méneville (Coleoptera: Curculionidae) atacando el tallo de árboles de aguacate en Colombia. Rev. U.D.C.A. Act. \& Div. Cient. 12(1):59-68.

https://doi.org/10.31910/rudca.v12.n1.2009.642

24. SERVICIO NACIONAL DE SANIDAD INOCUIDAD Y CALIDAD AGROELIMENTARIA, SENASICA. 2018. Manual de identificación de las principales plagas del aguacate en México. Tecámac, México. 32p.

25. VÉLEZ-ÁNGEL, R.; MADRIGAL CARDEÑO, J.A.; MORALES, G. 1982. Biología, hábitos y hospedantes del minador del crisantemo. Revista Colombiana de Entomología. 6:21-36.
26. WYSOKI, M.; VAN DEN BERG, M.A.; ISH-AM, G.; GAZIT, S.; PEÑA, J.E.; WAITE, G.K. 2002. Pests and pollinators of avocado. In: Peña, J.E.; Sharp, J.L.; Wysoki, M. Tropical fruit pests and pollinators: biology, economic importance, natural enemies and control. p.223-293.

27. YEFREMOVA, Z.A.; KRAVCHENKO, V.D. 2015. Interactions among host plants, lepidoptera leaf miners and their parasitoids in the forest-steppe zone of russia (Insecta: Lepidoptera, Hymenoptera). SHILAP Revista de Lepidopterología. 43(170):271-280.

28. ZENNER DE POLANIA, I. 1986. Guía general de manejo de plagas en el cultivo de la papa. Instituto Colombiano Agropecuario (Bogotá). 36p. 